\title{
Differential expression of tropomyosin 3 in human epithelial ovarian cancer.
}

Shahan Mamoor, MS ${ }^{1}$

$3 \quad$ shahanmamoor@gmail.com

East Islip, NY USA

Epithelial ovarian cancer (EOC) is the most lethal gynecologic cancer (1). We performed discovery of genes associated with epithelial ovarian cancer and of the high-grade serous ovarian cancer (HGSC) subtype, using published microarray data $(2,3)$ to compare global gene expression profiles of normal ovary or fallopian tube with that of primary tumors from women diagnosed with epithelial ovarian cancer or HGSC. We identified the gene encoding tropomyosin 3, TPM3, as among the genes whose expression was most different in epithelial ovarian cancer as compared to the normal fallopian tube. TPM3 expression was significantly higher in high-grade serous ovarian tumors relative to normal fallopian tube. TPM3 expression correlated with overall survival in patients with ovarian cancer. These data indicate that expression of TPM3 is perturbed in epithelial ovarian cancers broadly and in ovarian cancers of the HGSC subtype. TPM3 may be relevant to pathways underlying ovarian cancer initiation (transformation) or progression.

Keywords: ovarian cancer, epithelial ovarian cancer, HGSC, high-grade serous ovarian cancer, systems biology of ovarian cancer, targeted therapeutics in ovarian cancer. 
The five-year survival rate for women diagnosed with high-grade serous ovarian cancer is between $30-40 \%$ and has not changed significantly in decades $(4,5)$. The development of novel, targeted therapeutics to treat HGSC can be facilitated by an enhanced understanding of the transcriptional behavior of ovarian tumors relative to that of the normal ovary. We mined published microarray data $(2,3)$ to compare global gene expression profiles between human ovarian tumors, including that of the HGSC subtype, and that of normal ovarian and fallopian tissue. We identified the gene encoding tropomyosin 3, TPM3, as among the most differentially expressed in HGSC tumors of the ovary and in epithelial ovarian cancer broadly. TPM3 may be a gene of interest when prioritizing the study of target genes and pathways for the development of novel therapeutic interventions in epithelial ovarian cancer and specifically in high-grade serous ovarian cancers.

\section{Methods}

We used microarray data from datasets GSE124766 (2) and GSE146556 (3) for this differential gene expression analysis of epithelial ovarian cancer in conjunction with GEO2R. The Benjamini and Hochberg method of p-value adjustment was used for ranking of differential expression but raw p-values were used for assessment of statistical significance of global differential expression. Log transformation of data was auto-detected, and the NCBI generated category of platform annotation was used. GSE124766 (2) was generated using Agilent-014850 Whole Human Genome Microarray 4x44K G4112F with $n=3$ normal fallopian tube tissue (control) and $n=8$ for tumors from patients with high-grade serous ovarian cancer. GSE146556 (3) was generated using Affymetrix Human Gene 1.0 ST Array with $n=3$ for normal ovarian tissue and $n=40$ for tumors from patients with epithelial ovarian cancer: $n=26$ high-grade serous ovarian cancer; $n=3$ carcinosarcoma, $n=2$ clear cell, $n=1$ endometrioid, $n=1$ granulosa cell, $n=1$ immature teratoma, $n=4$ mixed, and $n=2$ mucinous; analysis was performed using platform GPL6244. GEO2R provides mRNA expression levels only for the top 250 most differentially expressed genes transcriptome-wide.

A statistical test was performed to evaluate whether TPM3 expression was significantly different when comparing normal ovarian tissue from control subjects and primary tumors from women diagnosed with HGSC using a two-tailed, unpaired t-test with Welch's correction. We used PRISM for all statistical analyses (Version 8.4.0)(455). For Kaplan-Meier survival analysis, we used the Kaplan-Meier plotter online tool (6) for correlation of TPM3 mRNA expression levels with overall survival (OS) in $n=1656$ ovarian cancer patients.

\section{Results}

We mined published microarray data $(2,3)$ to identify differentially expressed genes in epithelial ovarian cancer and specifically in high-grade serous ovarian cancer (HGSC), the type of ovarian cancer responsible for $70-80 \%$ of deaths resulting from the most lethal gynecologic malignancy.

TPM3 is differentially expressed in ovarian tumors from women diagnosed with HGSC

We identified TPM3 as among the genes whose expression was most different when comparing primary HGSC tumors to normal fallopian tube tissue (2) (Chart 1). When sorting all of the transcripts measured by microarray based on change in expression between HGSC and the normal fallopian tube, in this data set, TPM3 ranked 231 out of 40481 total transcripts (Chart 1), equating to $99.4 \%$ differential expression. Differential expression of TPM3 in HGSC tumors was statistically significant (Chart 1; $p=1.90 \mathrm{E}-05)$.

We analyzed a second microarray dataset (3) generated using normal ovarian tissues and tumors 
from women diagnosed with epithelial ovarian cancer (EOC) to determine whether differential expression of TPM3 could be observed in tumors from a different group of patients, which included HGSC tumors.

We found that TPM3 was differentially expressed in epithelial ovarian cancers broadly, but potentially present as a fusion transcript with ROS1 and/or NTRK1 (Chart 2). When sorting all of the transcripts measured by microarray based on change in expression between tumors from patients with epithelial ovarian cancers and the normal ovary, TPM3//ROS1//NTRK1 ranked 156 of 29088 total transcripts (Chart 2), equating to $99.5 \%$ differential expression. Differential expression of TPM3//ROS1//NTRK1 in EOC was statistically significant (Chart 2; $\mathrm{p}=5.18 \mathrm{E}-08$ ). TPM3 has been identified as a fusion transcript with ROS1 in non-small cell lung adenocarcinoma (NSCLC) (7).

TPM3 is expressed at significantly higher levels in HGSC when compared to the normal fallopian tubes.

We then extracted exact mRNA expression levels for the differentially expressed TPM3 transcript from both normal fallopian tube tissue and from epithelial ovarian tumors. TPM3 was expressed at significantly higher levels in high-grade serous ovarian cancers when compared to the normal fallopian tube (Figure 1; $\mathrm{p}=0.0045$ ). We calculated a mean fold change of 1.12 in TPM3 expression when comparing HGSC tumors to normal fallopian tissue, as TPM3 was expressed at $12.18 \pm 0.28$ arbitrary units $(\mathrm{AU})$ in the fallopian tubes but at $13.67 \pm 0.18 \mathrm{AU}$ in tumors of the ovary.

$\underline{\text { TPM3 tumor expression is correlated with survival outcomes in patients with ovarian cancer. }}$

We performed Kaplan Meier survival analysis (6) to evaluate whether TPM3 tumor expression correlated with survival outcomes in patients with ovarian cancer. TPM3 primary tumor expression was correlated with overall survival (OS) in patients with ovarian cancer, in the lower quartile (Figure 2).

TPM3 expression was a negative prognostic indicator in patients with ovarian cancer, in the lower quartile. While median OS was 50.97 months for ovarian cancer patients with low tumor expression of TPM3, median OS was 41 months for ovarian cancer patients with high tumor expression of TPM3 (Chart 3). Correlation of OS with TPM3 tumor expression in patients with ovarian cancer, in the lower quartile, was statistically significant (Figure 2: log rank p-value: 0.03; hazard ratio: 1.3 (1.02-1.66)).

TPM3 primary tumor expression was not correlated with overall survival in the lower tertile (Figure 2: $\log$ rank p-value: 0.13; hazard ratio: $1.19(0.95-1.48)$ ), in the upper tertile (Figure 2: $\log$ rank p-value: 0.93; hazard ratio: $1.01(0.81-1.25)$ ), or in the upper quartile (Figure 2: log rank p-value: 0.45; hazard ratio: $0.91(0.72-1.16))$.

Thus, we found using published microarray data $(2,3)$ that TPM3 was among the genes most differentially expressed in tumors from women with high-grade serous carcinomas, and in epithelial ovarian cancer broadly. TPM3 expression was significantly higher in HGSC tumors when compared to the normal fallopian tubes, and TPM3 tumor expression was correlated with overall survival in patients with ovarian cancer.

\section{Discussion}

Transcriptional profiling of HGSC in a Cancer Genome Atlas integrated genomic analysis classified HGSC into four subtypes based on gene clustering: immunoreactive, differentiated, proliferative, and mesenchymal (8). We sought to describe the transcriptional landscape of epithelial ovarian cancers in an unbiased fashion by using published microarray data from primary tumors of women diagnosed with HGSC compared to transcriptome data from normal ovarian and fallopian tube tissue (2, $3)$. In both datasets analyzed (2, 3), TPM3 was among the genes whose expression changed most 


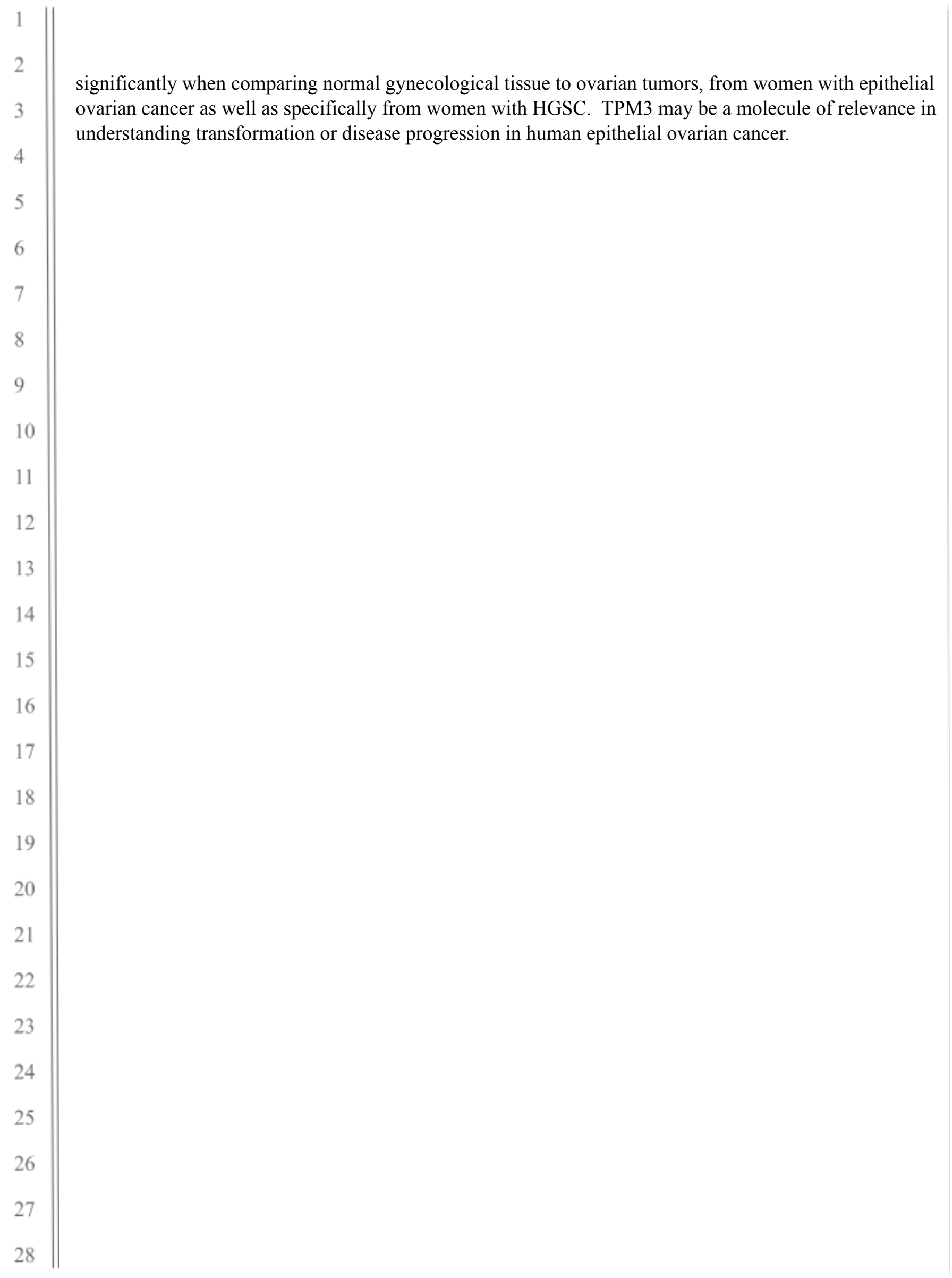




\section{References}

1. Guppy, A.E., Nathan, P.D. and Rustin, G.J., 2005. Epithelial ovarian cancer: a review of current management. Clinical Oncology, 17(6), pp.399-411.

2. Zhang, W., Klinkebiel, D., Barger, C.J., Pandey, S., Guda, C., Miller, A., Akers, S.N., Odunsi, K. and Karpf, A.R., 2020. Global DNA hypomethylation in epithelial ovarian cancer: passive demethylation and association with genomic instability. Cancers, 12(3), p.764.

3. Hoffmann, K., Berger, H., Kulbe, H., Thillainadarasan, S., Mollenkopf, H.J., Zemojtel, T., Taube, E., Darb-Esfahani, S., Mangler, M., Sehouli, J. and Chekerov, R., 2020. Stable expansion of high-grade serous ovarian cancer organoids requires a low-Wnt environment. The EMBO journal, 39(6), p.e104013.

4. Bowtell, D.D., Böhm, S., Ahmed, A.A., Aspuria, P.J., Bast Jr, R.C., Beral, V., Berek, J.S., Birrer, M.J., Blagden, S., Bookman, M.A. and Brenton, J.D., 2015. Rethinking ovarian cancer II: reducing mortality from high-grade serous ovarian cancer. Nature reviews Cancer, 15(11), pp.668-679.

5. Vaughan, S., Coward, J.I., Bast, R.C., Berchuck, A., Berek, J.S., Brenton, J.D., Coukos, G., Crum, C.C., Drapkin, R., Etemadmoghadam, D. and Friedlander, M., 2011. Rethinking ovarian cancer: recommendations for improving outcomes. Nature Reviews Cancer, 11(10), pp.719-725.

6. Győrffy, B., Lánczky, A. and Szállási, Z., 2012. Implementing an online tool for genomewide validation of survival-associated biomarkers in ovarian-cancer using microarray data from 1287 patients.

Endocrine-related cancer, 19(2), pp.197-208.

7. Takeuchi, K., Soda, M., Togashi, Y., Suzuki, R., Sakata, S., Hatano, S., Asaka, R., Hamanaka, W., Ninomiya, H., Uehara, H. and Choi, Y.L., 2012. RET, ROS1 and ALK fusions in lung cancer. Nature medicine, 18(3), pp.378-381.

8. Cancer Genome Atlas Research Network, 2011. Integrated genomic analyses of ovarian carcinoma. Nature, 474(7353), p.609. 


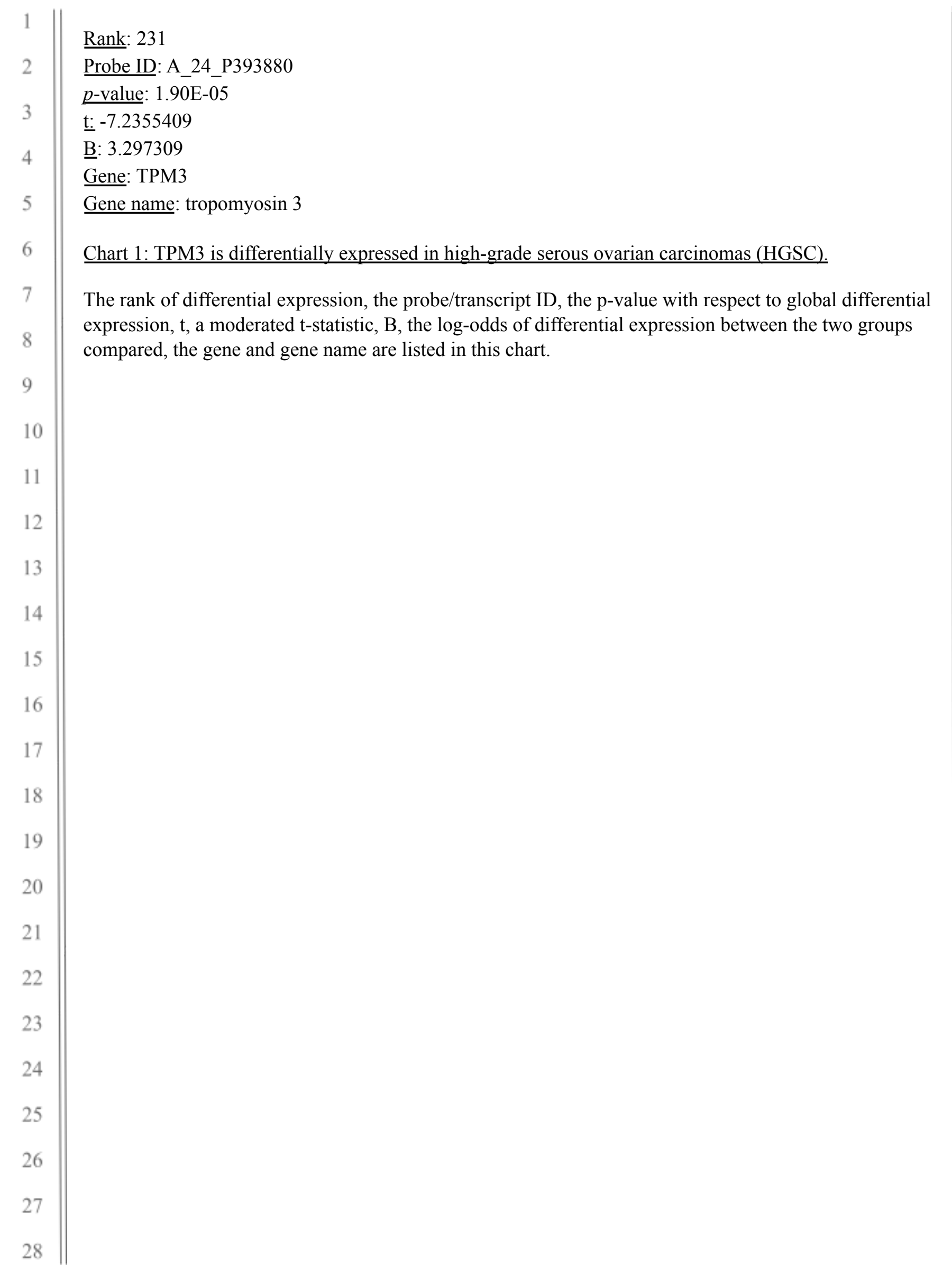




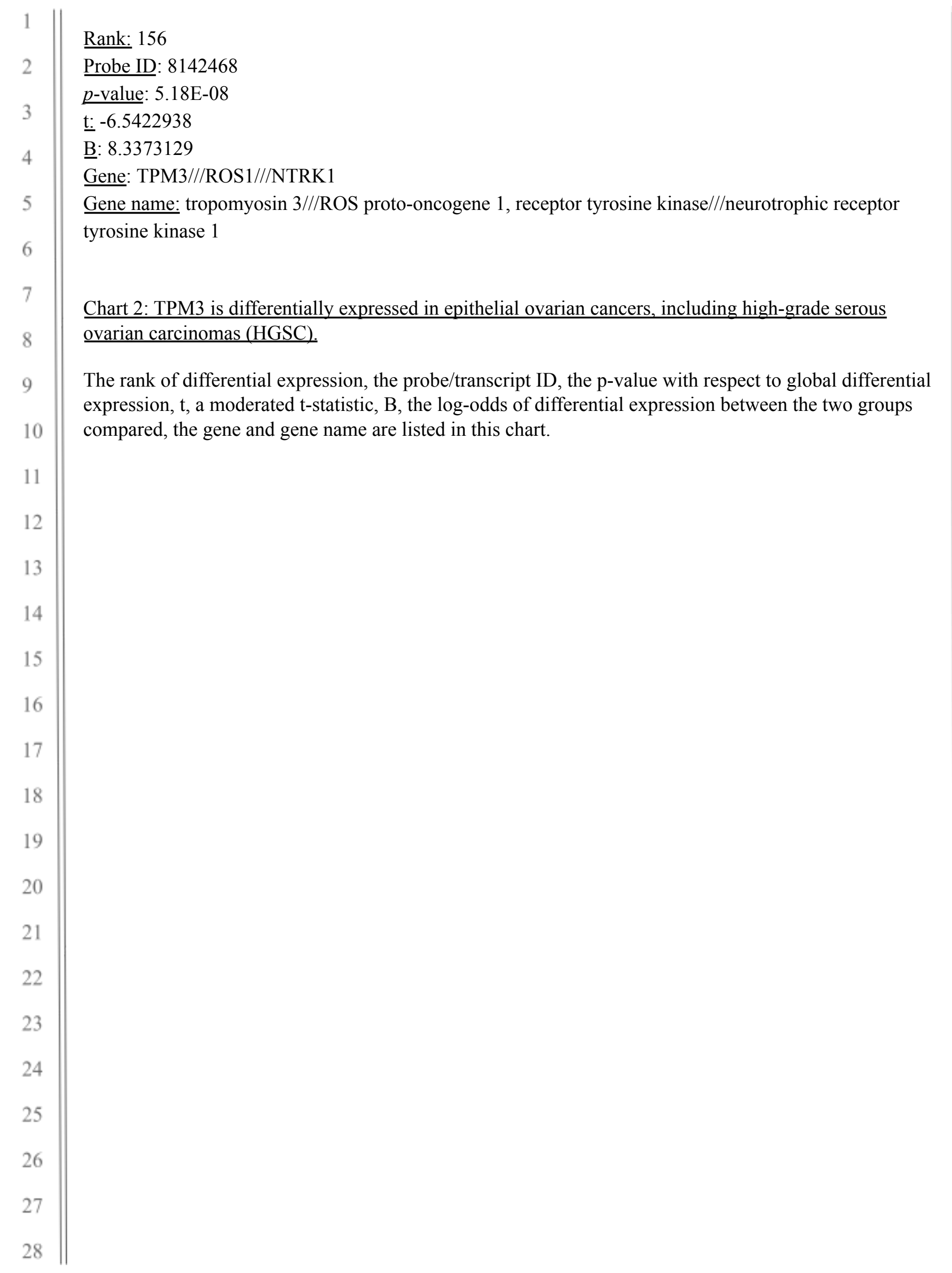




\section{TPM3}

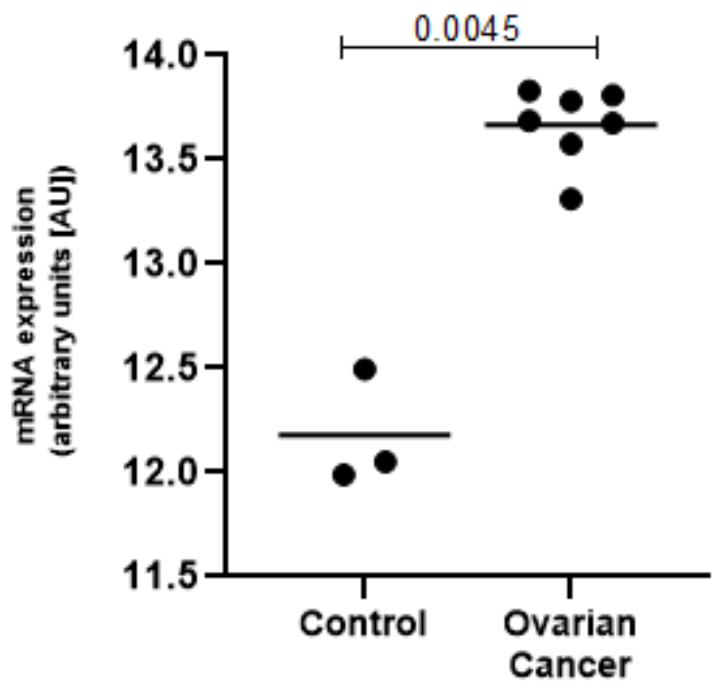

Figure 1: TPM3 is expressed at significantly higher levels in HGSC tumors when compared to normal fallopian tube.

The mRNA expression of TPM3 in normal fallopian tube tissues (left) and in the primary tumors of patients with HGSC (right) is represented with mean mRNA expression level marked and the result of a statistical test evaluating the significance of difference in TPM3 expression between the normal fallopian tube and primary tumors from patients with HGSC, a $p$-value, listed above. 


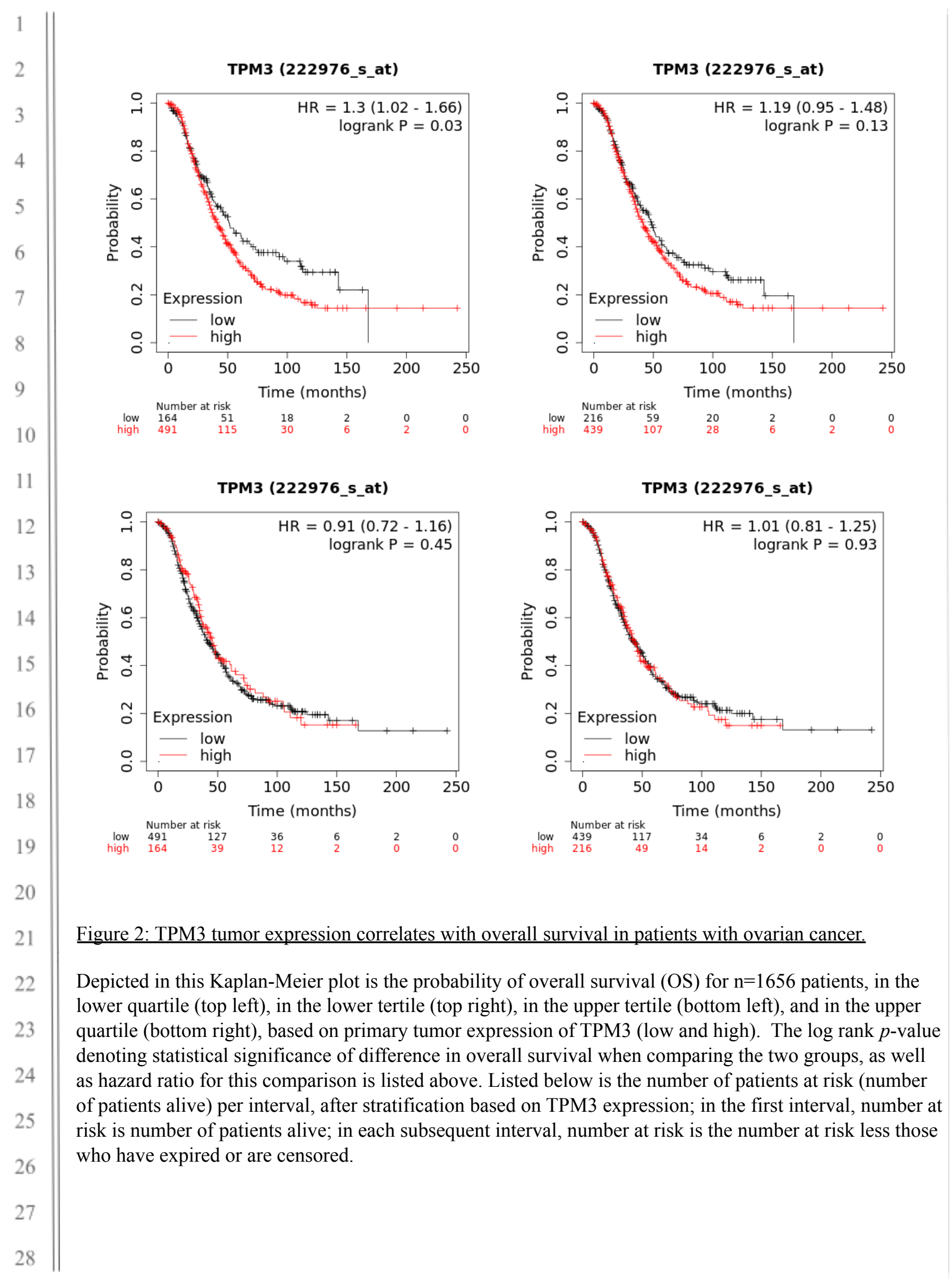




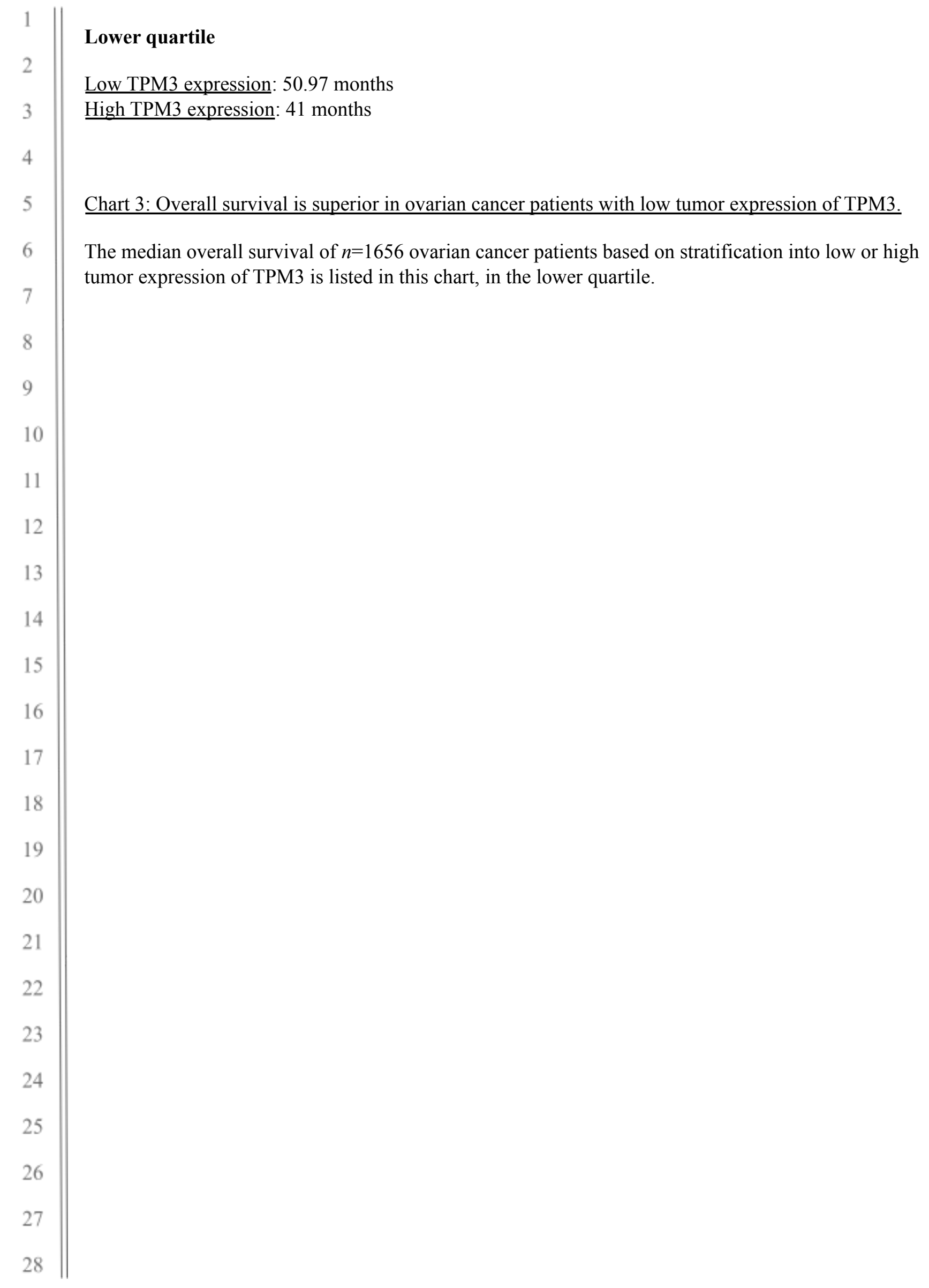

